\title{
Der Palmengarten und die „Freunde des Palmengartens“ auf dem Fürstlichen Gartenfest 2013 auf Schloss Wolfsgarten
}

\author{
Lolita Sebastian
}

\section{Immer dabei}

Vom 20.-22. September 2013 fand zum 7. Mal das Fürstliche Gartenfest auf Schloss Wolfsgarten in Langen statt. Auf dieser vom Landgrafen von Hessen ausgerichteten hochwertigen Gartenmesse gibt es neben vielen Verkaufsständen mit Pflanzen und Gartenasseccoires, Schaugärten und einem reichhaltigen kulinarischen Angebot auch diverse Informationen rund um Gartenthemen. Jedes Jahr wird ein anderes Sonderthema ausgelobt, diesmal stand „English Gardening" auf dem Programm. Wie immer zeigte sich das Wetter in diesen Herbsttagen von seiner besten Seite, morgens wallten die Nebel, sodass überall mit Tröpfchen verzierte Spinnennetze den Garten zierten. Und wie jedes Jahr waren der Palmengarten und die „Freunde“ mit einem gemeinsamen Informationsstand vertreten.

\section{Vorbereitungen von langer Hand}

Schon Wochen vor der Veranstaltung wurden Vorbereitungen getroffen und Ideen gesammelt, wie der Stand gestaltet wird, welche Exponate gezeigt werden und wie das Sonderthema umzusetzen ist. Vor allem Jörg Kunz und Dirk UllRich legten Hand an, besorgten Pflanzen für die Dekoration und setzten ihre Gestaltungsideen sehr kreativ um. Allein für die geplanten herbstlichen Gestecke wurden 750 Chrysanthemen benötigt. Um mit Besucherinnen und Besuchern des Gartenfestes ins Gespräch zu kommen und dabei auf den Palmengarten aufmerksam zu machen, wurden allerlei Zapfen, Früchte, Hölzer und andere interessante botanische Objekte für unsere Theke ausgewählt. Auch die mit bunten Früchten und Samen gefüllten Setzkästen kamen wieder zum Einsatz und bewirkten Staunen über die bota-

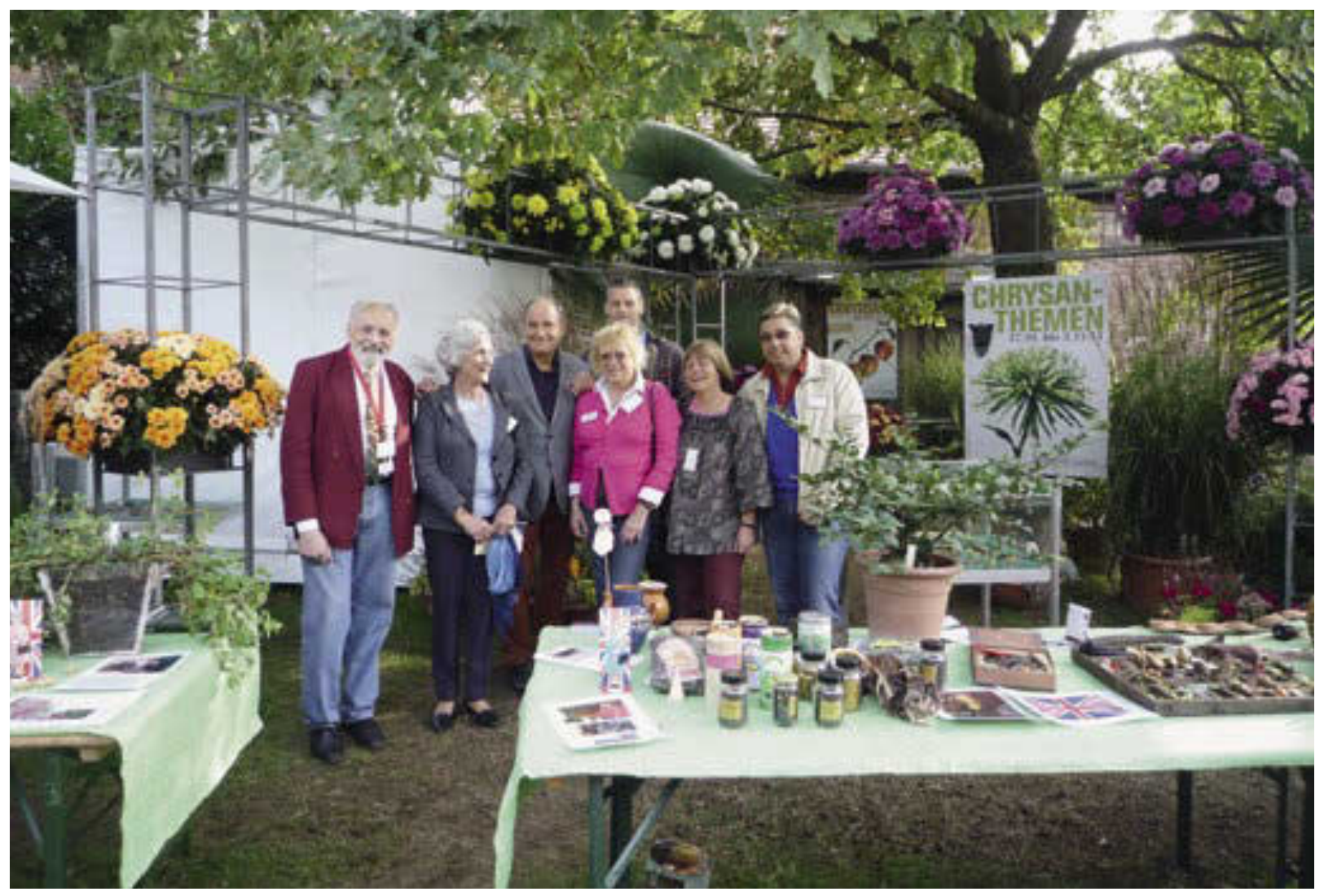




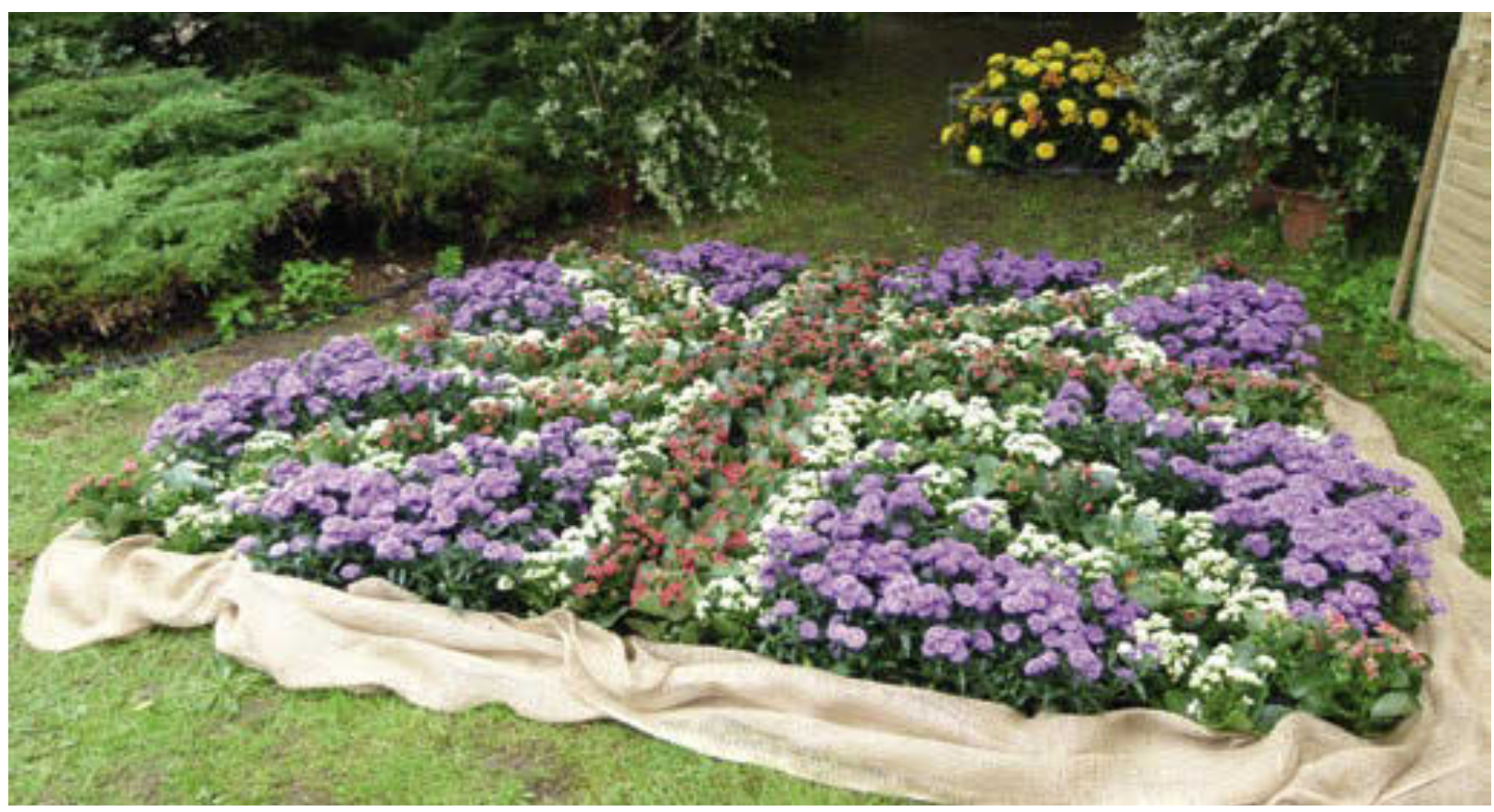

nische Vielfalt. Dazu kamen dann noch der Präsentationswagen und das Werbematerial der „Freunde“. Jede Menge Jahres- und Monatsprogramme sowie Informationsflyer haben wir in den drei Tagen unter das Volk gebracht. Außer der Autorin, der Schatzmeisterin der „Freunde“, waren Herbert Billensteiner, Jörg Kunz, Hilke Steinecke und Dirk Ullrich mit von der Partie. Am Freitag unterstützten uns Conny Schaan und Astrid Steindorf.

\section{Buntes Treiben}

Die Messeleitung achtet darauf, dass Aussteller von Jahr zu Jahr nicht immer den gleichen Standplatz erhalten, damit sich während der Feste stets ein etwas anderes Bild ergibt. Diesmal hatten wir einen besonders exponierten Platz unterhalb des Messebüros an einem Durchgang zum Hauptweg. Mit einer alten Eiche und einer Bruchsteinmauser im Hintergrund, an die eine alte Holzhütte angebaut war, fühlten wir uns auf unserem Standplatz fast wie in einem cottage garden. Dem Sonderthema „English Gardening“ wurden wir gerecht, indem Jörg KunZ und Dirk UllRich auf einem Teil unserer Fläche die englische Flagge aus blauen Astern sowie weißen und roten Kalanchoen gestalteten. Verschiedene Teesorten und Exponate rund um das Genussmittel Tee er-

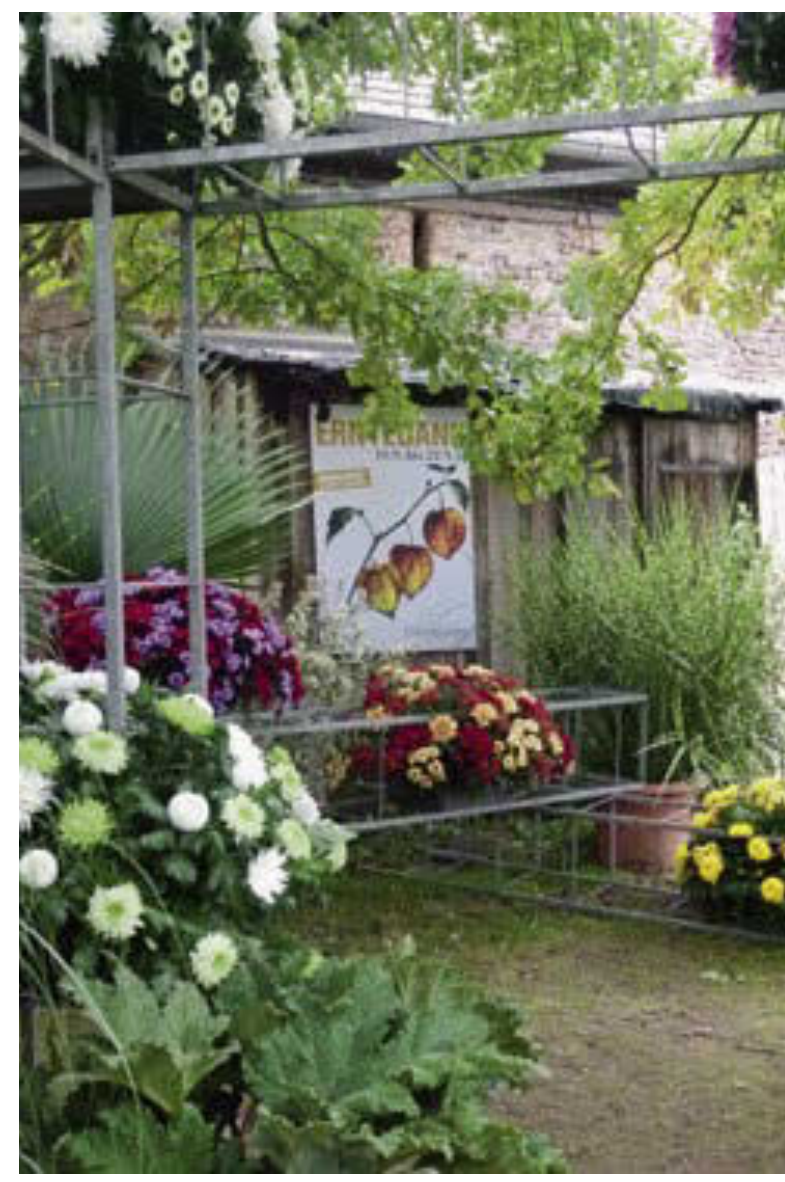

Abb. 1 (Seite 154): Viel Spaß am Stand des Palmengartens.

Abb. 2 (oben): Britische Flagge aus Blüten.

Abb. 3 (unten): Herbstliche Dekoration mit farbenfrohen Chrysanthemen. 


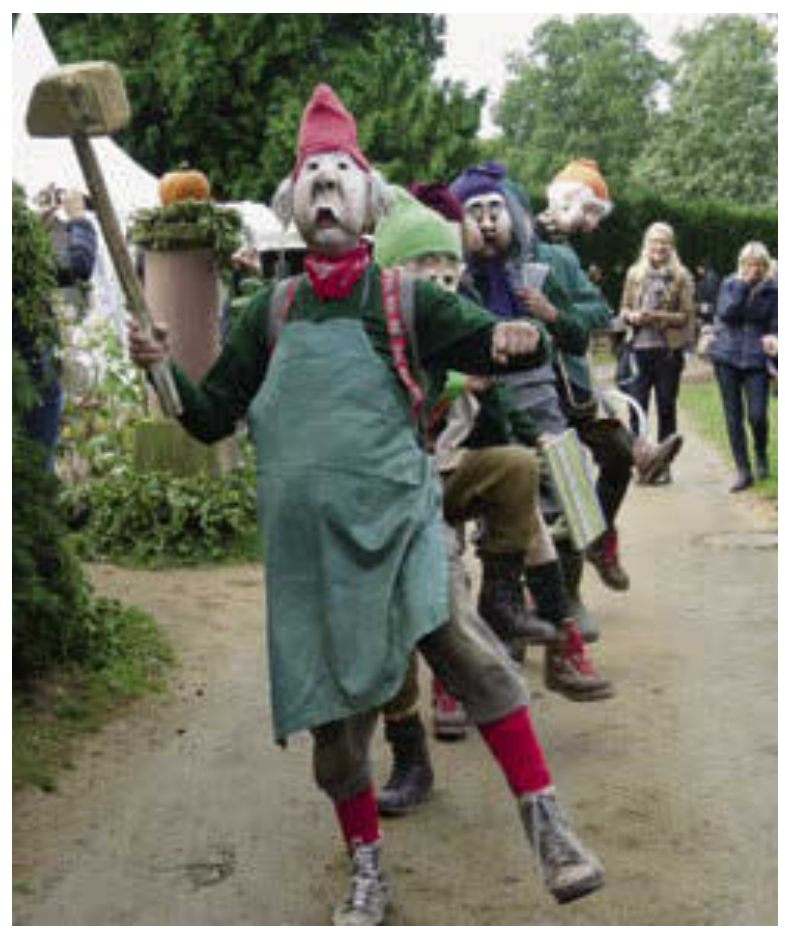

gänzten das „English Gardening“. Eine rosa und blaue, solarbetriebene winkende Queen zauberte allen Vorbeikommenden immer wieder ein breites Lächeln ins Gesicht. Die Besucher kamen in Scharen und fragten uns zu den Ausstellungsobjekten Löcher in den Bauch. Auch die Schatzmeisterin der Freunde des Palmengartens lernte so langsam das eine oder andere "Botanische“" und brachte das neu erworbene Wissen gerne unter die Leute. Viele Bekannte kamen vorbei und freuten sich darüber, auf dem Fürstlichen Gartenfest auch den Palmengarten anzutreffen. Dabei hatten wir auch die Gelegenheit, Holger Weinert vom Hessischen Rundfunk zu begrüßen. Besuch erhielten wir zudem von der Vorsitzenden der Freunde des Palmengartens. Prinzessin von Hannover unterstützte uns bei der Werbung für den Palmengarten und den Verein. Als am Samstag Hilke Steinecke vor Ort einen Vortrag über die Geschichte des Tees hielt, war unser Stand dicht mit Interessenten gefüllt.

\section{Erfolgreiches Fest}

Wie auch in den Vorjahren war Hilke SteinECKE am Freitagnachmittag mit der Botanikpreis-Jury unterwegs, um die Empfänger des goldenen, silbernen und bronzenen Wolfskop-

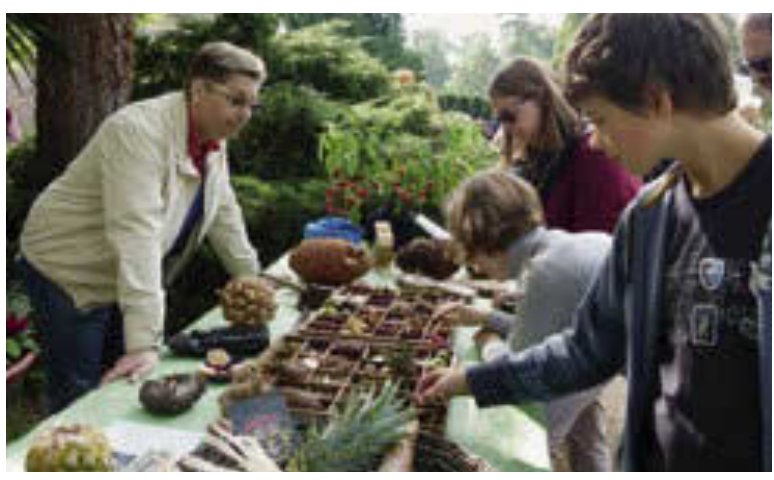

fes sowie den Gewinner des Landgraf-vonHessen-Preises zu ermitteln. Wir selbst hatten uns um den Pressepreis beworben. Wir hatten Erfolg und erhielten für unseren Stand einen bronzenen Wolfskopf. Als während des Ausstellerabends die Preise verliehen wurden und Jurymitglied Holger Weinert seine Laudatio mit den Worten '..und die winkende Queen ...' begann, war uns klar, dass wir den bronzenen Pressepreis gewonnen hatten.

Drei Tage Wolfsgarten zuzüglich Auf- und Abbau haben viel Freude gemacht und das Teamgefühl gestärkt, waren aber auch sehr anstrengend. Mit Sicherheit haben wir vielen Besucherinnen und Besuchern wieder in Erinnerung gerufen, doch in den Palmengarten zu kommen. Und möglicherweise wird der eine oder andere Mitglied bei den „Freunden“. Am Ende der Veranstaltung wurden die farbenfrohen Chrysanthemen aus den Gestecken gegen eine Spende an die Vorbeikommenden weitergegeben. Die bepflanzten Schalen wurden in den Palmengarten zurückgenommen und zierten noch Wochen nach dem Fest die Gartenseite des Eingangsschauhauses. Auch die Flagge wurde für etwa drei Wochen vor dem Eingangsoktogon wieder aufgebaut.

Noch Wochen nach der Veranstaltung begrüßten wir uns gegenseitig mit dem typischen royalen Winkegruß unserer Solar-Queen. Wir haben beschlossen: Nächstes Jahr sind wir wieder dabei.

Abb. 4: Die sieben Zwerge kommen am Palmengarten-Stand vorbeimarschiert.

Abb. 5: Großer Andrang botanisch Interessierter. 